\title{
Protée
}

\section{L’ombilic de Dieu. Le fou et le cabaliste}

\section{Anne Élaine Cliche}

Volume 28, numéro 1, 2000

\section{Variations sur l'origine}

URI : https://id.erudit.org/iderudit/030584ar

DOI : https://doi.org/10.7202/030584ar

Aller au sommaire du numéro

Éditeur(s)

Département des arts et lettres - Université du Québec à Chicoutimi

ISSN

0300-3523 (imprimé)

1708-2307 (numérique)

Découvrir la revue

\section{Citer cet article}

Cliche, A. É. (2000). L’ombilic de Dieu. Le fou et le cabaliste. Protée, 28(1), 59-74. https://doi.org/10.7202/030584ar
Résumé de l'article

L'auteur fait ici une relecture du cas Schreber et de ses troublants rapports à Dieu. Le Dieu de Schreber est un dieu de langage, et le système complexe de rayons et de nerfs qui le rattache à Schreber a une consistance verbale. Cette merveilleuse construction vient répondre à une origine défaillante entrevue par le psychotique comme un trou sans bord provoquant l'effondrement des mondes. L'analyse compare cet aspect du système schreberien avec la logique du cabaliste Abraham Aboulafia et son émanation du « dibbour », projection et contemplation des lettres de l'alphabet hébraïque comme ombilic de Dieu. Le fou et le cabaliste se rencontrent sur cette question de l'origine. 


\section{L'O M BILIC DE DIEU LE FOU ET LE CABALISTE*}
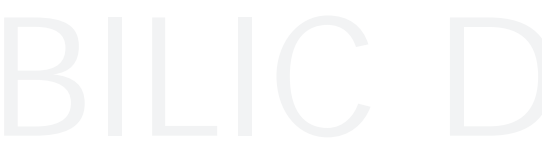

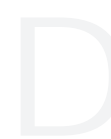

ANNE ÉLAINE CLICHE

Aux étudiants du séminaire "Délire et théorie» donné à l'automne 1999, qui m'ont aidée à élaborer plusieurs aspects de cette analyse par leur patiente écoute et leurs commentaires judicieux.

L'argument selon lequel, au temps des périodes glaciaires, l'humanité n'en était qu'à ses tout premiers commencements (diluviens) n'est guère convaincant. Qui nous prouve qu'il n'y a pas eu dès cette époque reculée sur une certaine planète, et je penche pour Vénus, une humanité hautement développée, dont l'anéantissement entrait dans les plans de Dieu [...]. D. Paul Schreber ${ }^{1}$

Tout comme le Midrash la cabale enseigne qu'avant la création de nos mondes d'autres furent créés et ensuite détruits. Georg Langer ${ }^{2}$

\section{AU DÉBUT EST LE PARLER}

Beréchit, au commencement... Le mot hébreu qui ouvre la Bible n'est pas un mot comme les autres. Il occupe dans la tradition juive une place singulière que les traductions ne sauraient que... traduire mais non restituer en langues des nations. Le mot, en effet, est unique et n'apparaît qu'à cette place inaugurale du Livre. On le retrouve seulement dans Jérémie où il prend un autre sens puisqu'il se construit avec le mot suivant - «Au commencement du règne de Yehoyakim». Rachi, le grand commentateur de la Torah au XIe siècle, ira dès lors jusqu'à affirmer qu'il faudrait lire, de même: «Au commencement de l'acte de la création où Dieu créa le ciel et la terre" ${ }^{3}$. Autrement dit, la sagesse talmudique avance qu'il ne saurait s'agir là d'un commencement absolu présentant du même coup l'ordre d'apparition du monde, mais d'un commencement particulier, celui où Dieu créa le ciel et la terre. En effet, le premier hébraïsant venu vous dira que be-réchit n'équivaut pas tout à fait à la formule "Au commencement», mais se traduirait plutôt par «En un commencement", l'article défini manquant à sa place. Ce qui, avouons-le, change un peu la perspective. Qu'il y ait eu d'autres commencements ne suscite d'angoisse chez aucun familier de la sagesse juive, et le mot a pour conséquence de rappeler que le commencement ne se confond pas avec l'origine qui, elle, relèverait plutôt d'une mise en place ultérieure, d'un surgissement après coup et sur le mode mythique pour désigner la cause et non ce qui d'elle s'actualise. 
Beréchit est un mot forgé, créé pour ouvrir le Livre. Il se compose de la préposition be (dans), du nom rosh (tête) et de la désinence it qui marque le caractère abstrait du terme. La langue hébraïque est une langue à racines bi et tri-litères, ce qui implique une sorte d'étymologie toujours en acte dans la parole, ou si l'on veut, une origine présente dans le verbe, et dont l'actualisation est en quelque sorte latente, toujours prête à servir le sens. Ainsi ReCHiT vient de ROCH, trois lettres en hébreu, d'où l'on peut traduire «Entête», comme le fait Chouraqui ${ }^{4}$. Par ce mot, le statut du commencement est donc à l'avance déplacé et démultiplié, ramené au mot pour le dire qui est le premier et qui commence le Livre, faisant se conjoindre l'entête du Livre et l'entête de la création, affirmant d'emblée que le monde en question ne commence qu'avec le Livre. Ce que la tradition juive ne cesse d'ailleurs de marteler dans tous ses registres d'interprétation. Quant à la cabale, on va le voir, elle saura tirer un profit singulier de cette inscription.

Mais il y a plus. Que le mot soit la chose, que la parole crée le monde - ce que l'histoire du Livre Entête ou Genèse raconte et met en scène - se révèle en cette tradition n'être pas seulement le privilège de Dieu mais la vertu de la langue elle-même. Car la langue hébraïque entretient avec le monde des rapports étranges, franchement étonnants; rapports que la rationalité linguistique ne saurait ni simplifier ni réduire sainement à l'arbitraire du signe. La raison hébraïque est avant tout motivée par le nombre, entendez par les lois de la combinatoire, dont la fonction n'est pas tant de représenter que de rendre au sens sa dynamique d'engendrement. Il faudrait déjà entendre ici, et sans s'émouvoir, que la motivation est en cette langue un facteur sérieux de signification.

Il y a bien sûr, dans l'hébreu, une structure qui le lie à toutes les langues, structure qui renvoie chaque phonème à un autre ordre que lui et ce, de manière contingente. On se souvient ici de Saussure (1969: 126) qui, par «arbitraire», voulait dire que le signe demeure «immotivé par rapport au signifié, avec lequel il n'a aucune attache naturelle dans la réalité».
Débat enfin tranché entre Cratyle et Hermogène, entre la nature et la convention, et qu'on ne saurait rouvrir sans tomber une fois de plus dans les impasses trop connues. Même si les textes littéraires et les matériaux inconscients ne cessent de révéler le déterminisme de la matière même du signe, la notion saussurienne d'arbitraire reste incontestable et frappe de contingence toutes les langues alphabétiques sans exception. On ne peut pas parler l'hébreu sans soutenir, la reconnaissant ou non, cette condition linguistique première. Pourtant, la tradition juive - suivant ses lois d'interprétation et de transmission ne l'entend pas de cette oreille. La langue, en cette culture, est d'essence divine. Qu'est-ce à dire, sinon que son origine, sa provenance, si elle demeure incontestablement historique, est aussi le fait de Dieu: création dont la matière est beréchit, non conventionnelle, adéquate à l'acte qui est commencement simultané du verbe et du monde, du mot et de la chose (en hébreu, d'ailleurs, le mot davar signifie à la fois parole et chose)?

La cabale n'est qu'une exploitation poussée de cette conception juive de la langue. «En hébreu, une lettre, oth, est aussi un signe et il n'y a pas d'arbitraire du signe, en ce sens que la forme de chaque lettre et sa dénomination ont des significations en rapport l'une avec l'autre» (Atlan, 1982: 57). L'hébreu, suivant cette logique, n'est pas seulement une langue alphabétique mais un système de hiéroglyphes ou de «substances dont les lettres sont les éléments». La mystique va tirer toutes les conséquences de cette tradition.

Car, pour la tradition mystique juive, non seulement le signe est motivé et désigne le réel sans laisser de résidu, mais le signifiant est lui aussi une incarnation du signifié, c'est-à-dire que le niveau phonématique est déjà significatif, alors que pour la science linguistique il n'est que distinctif-oppositif. Une telle conception débouche inévitablement sur un "étymologisme» qui considère les graphèmes ou/et les phonèmes comme des hiéroglyphes. Il y aurait comme une hiéroglyphisation de la langue hébraïque. (Banon, 1987: 182-183)

À cela, on rétorquera sans doute qu'il faut être croyant, mystique ou fou pour soutenir la divinité de 
la langue. Nous devrons donc provisoirement en passer par là. Mais il se peut qu'au sortir de ce parcours par la croyance, la mystique et la folie, la notion de signifiant s'en trouve éclairée; ce qui ne serait pas une mince contribution. Souvenons-nous seulement, pour commencer, que l'athéisme ne consiste pas à se débarrasser de Dieu. Tâche impossible s'il en est. Car Dieu, plus il est mort, plus il existe, toute la question, bien sûr, étant de connaître sa nature. Détail dont nous nous occupons ici.

\section{SCÈNE PRIMITIVE DU MONDE}

Un homme, un seul homme, n'aurait-il pu être alors sauvé pour

la conservation de l'espèce - celui peut-être qui eût été de mours plus méritantes relativement? - et cet homme, les voix qui me parlent me le désignent sous le nom de Der ewige Jude. (53)

Le livre de Daniel-Paul Schreber, Denkwürdigkeiten eines Nervenkranken ${ }^{5}$, qu'il faudrait traduire, nous diton, par «Les hauts faits ou la geste mémorable d'un malade des nerfs " ${ }^{6}$, ne préoccupe sans doute pas seulement les psychiatres et les psychanalystes. Il risque d'intéresser quiconque a des rapports troublants avec la langue; autant dire, les écrivains. On trouve en effet dans ce livre qui s'écrit à la fois comme un témoignage, une plaidoirie, une épopée autobiographique et une théologie, le récit d'un homme aux prises avec des forces essentiellement parlantes. D'où vient cette parole incessante et multiple? Tout le livre de Schreber se compose - d'ailleurs admirablement - pour nous renseigner sur cette affaire. Il s'agit, nous informe le malade, de Dieu lui-même auquel - Schreber est un homme des Lumières - je ne crois pas d'emblée. Pourtant, l'accession à ce qui est raconté exigera de nous, affirme l'auteur, un acte de foi: «L'homme doit s'habituer à ce qu'il y ait des choses qui soient, en vérité, bien qu'il ne puisse pas les comprendre»(2). Quant au témoin luimême, sa parole ne relève ni de la croyance ni de la conversion; c'est de révélation qu'il s'agit?

La révélation est en effet fulgurante; et la catastrophe, radicale. Si l'on résume très vite l'histoire de cette geste avant tout clinique, on se souviendra d'abord que Daniel-Paul Schreber a été malade puis soigné par le docteur Flechsig pendant l'année 1883. 1884. Cette première affection qualifiée d'hypocondrie se déroula, nous dit Schreber, «sans les moindres incidents touchant le domaine surnaturel» (35). La guérison apparente dure plus de huit ans pendant lesquels son bonheur conjugal est assombri par la déception répétée d'un désir d'enfant. En juin 1893, Schreber apprend sa nomination au poste prestigieux de Président de la Cour d'appel, et entre en fonctions le $1^{\text {er }}$ octobre suivant. Entre ces deux dates se situent des rêves importants dans lesquels sa maladie nerveuse antérieure fait retour. Enfin, s'impose à lui, entre sommeil et veille, «la représentation que, tout de même, ce doit être une chose singulièrement belle que d'être une femme en train de subir l'accouplement" (36). À la fin d'octobre 1893, une insomnie torturante le ramène à la clinique de Flechsig.

Ce que l'auteur nous raconte dans ses Mémoires concerne cette «seconde" maladie qui le conduit à se prêter de nouveau aux traitements du conseiller privé et professeur Flechsig, alors qu'il est en pleine perturbation hallucinatoire d'où il va peu à peu se reconstituer, délirant sans relâche, mais suivant des transformations qu'il se fait un devoir de nous souligner de manière minutieuse et dramatique. La période la plus obscure de la psychose est sans doute celle qui remonte à cette époque de la maladie et qui, dans le livre dont la composition s'entreprend en 1900, se présente comme un souvenir, avec, au dire de Schreber lui-même, les reconstructions et les distorsions qui s'imposent (65). L'état crépusculaire du sujet constitue alors le noyau d'une vision bouleversante de fin du monde qui, par le voyage à rebours du temps qu'elle entraîne, vient inscrire en quelque sorte une scène primitive de l'humanité. Cette expérience visionnaire, qui inaugure et semble mettre en abyme la descente aux enfers de la folie, appartient pour Schreber à ce qu'il appelle un temps sacré (heilige Zeit).

Je puis le dire: le temps que j'ai essayé de dépeindre [...] (à peu près de la mi-mars à la fin mai 1894, s'il faut admettre qu'il s'est réellement agi là de mois terrestres et non pas de siècles) a 
certainement été la période la plus atroce de ma vie. Pourtant, ce temps fut aussi le temps sacré de ma vie, celui pendant lequel, mon âme, tout exaltée des choses surnaturelles qui m'envahissaient toujours plus abondamment au milieu du rude traitement que j'endurais de l'extérieur, était remplie des représentations les plus sublimes sur Dieu et l'ordre de l'univers. (63)

Ce temps sans mesure est l'une des premières conséquences de la «relation désormais indissoluble» (70) entre Dieu et Schreber dès lors frappé, sinon foudroyé, par des visions apocalyptiques dans lesquelles s'exprime l'engloutissement des mondes et de l'humanité entière, rendant caduque toute volonté de prise de notes pour une postérité devenue impensable; «[...] je croyais l'humanité tout entière engloutie; par conséquent prendre des notes n'aurait eu aucun sens" (65). Des trois visions racontées au chapitre VI des Mémoires, je retiendrai la première, qui s'apparente à une traversée géologique de l'histoire et semble devoir aboutir à l'origine de l'humanité, à cette place ombilicale de la terre-mère, approchée dans l'expérience comme le point le plus redoutable. Cette place originaire prend, dans la vision de Schreber, la forme d'un effondrement, d'un puits catastrophique sans bords ni revers, dont on ne peut que s'éloigner avec la certitude d'une déperdition irrémédiable.

[...] assis dans un compartiment de chemin de fer, ou dans un ascenseur, je descendais dans les profondeurs de la terre et je parcourais pour ainsi dire toutes les couches de l'humanité sinon de la terre; dans les régions supérieures, il y avait encore des forêts à essences feuillues; il faisait de plus en plus obscur et de plus en plus noir dans les régions profondes. Lorsque par moments je me risquais hors du vaisseau, je me promenais comme dans un vaste cimetière et, parmi ces lieux où reposait la population de Leipzig, mon chemin croisait la tombe de ma propre femme. Remonté dans le vaisseau, je m'arrêtais en un certain point $n^{0} 3$; je redoutais de devoir franchir le point $n^{0} 1$ qui marquait les débuts les plus reculés [Uranfang] de l'humanité. Au retour le puits [Schacht] s'effondra [stürzte] derrière moi, menaçant de façon permanente un certain «Dieu du soleil» qui s'y trouvait également. À ce propos il était dit qu'il y avait deux puits (correspondant au dualisme des Royaumes divins?). Lorsque parvint la nouvelle que le deuxième puits s'était effondré [eingestürzt sei], on crut que tout était perdu. (74)

La butée sur l'Uranfang, on l'entend, est éprouvée à la fois au futur et au passé, à jamais infigurable, sauf «au retour» (Rückwärtsfahren: mouvement de marche arrière), par la voix d'une «nouvelle». Le sujet est ainsi informé d'une menace et d'un désastre auxquels il assiste littéralement par ouï-dire. Que sont ces puits (Schächte: puits de mine) qui s'effondrent (stürzen) sinon des ouvertures béantes s'écroulant sur ellesmêmes, sans suture ombilicale envisageable, entraînant à leur suite un déferlement qui semble anéantir toute limite? Cette «vision» se retrouve encore dans l'«idée d'un grand trou dans le temps» (85) (großen zeitlichen Kluft) qui vient à l'esprit du Président pour nommer l'abîme creusé dans l'histoire de l'humanité et apparemment traversé par lui en une période irrepérable nommée «temps du premier jugement de Dieu " (83), trou encore une fois sans bords, marqué par «l'extinction des horloges du monde»; béance temporelle qui entraîne simultanément «un afflux continu et inhabituellement abondant de rayons en direction de mon corps" (85). Mais cette certitude d'un gouffre originaire occupe surtout les premières pages du livre qui racontent sur le mode épique l'avènement de «la faille» (Riß: déchirure, lézarde, crevasse) liée aux «destinées personnelles» du sujet, et qui permet de dire la crise à l'origine de sa folie (22): cet événement obscur d'un «meurtre d'âme originaire» ayant perturbé l'ordre de l'univers qu'il est désormais, lui, Schreber, contraint de restaurer. La notion de "Premier jugement de Dieu», assez étonnante puisqu'elle reporte au commencement un jugement qui d'ordinaire est dernier, vient donc couvrir l'abîme telle une procédure judiciaire à répétition engendrant une série de jugements dont l'objet demeure le conflit entre Flechsig et Schreber.

On remarque qu'il y a, dans la vision grandiose du voyage en véhicule tout-terrain (train-ascenseurvaisseau), l'expression d'un franchissement que les points 1, 2 et 3 viennent scander suivant un 
mouvement d'avant-arrière (ou d'aller-retour) selon lequel ce qui compte n'est pas l'aboutissement mais le «retour» informé. Comme si l'origine ne pouvait se dire qu'en cette construction - effondrement - à laquelle le corps ne saurait assister mais dont il doit prendre acte comme de sa causalité singulière. À la béance, qui s'ouvre et déferle vers lui, répondra ce corps, entièrement occupé à la fabrique d'une suture, qui recueillera les rayons et fils permettant de tisser, en un système de nœuds complexe, l'ombilic du monde; corps désigné à cette tâche puisque c'est en sa lignée que la crise a eu lieu, que c'est son nom, Schreber (celui de son ancêtre), qui fut assassiné par l'âme Flechsig dans ce combat ancien qui a tout déclenché. Lélection est ici portée au registre qui convient, et le corps à corps avec Dieu n'est encore que la forme imposée par l'«effondrement» nerveux et spirituel (Nervenstürz et Zusammenbruch) éprouvé comme conséquence d'une crise de l'origine.

Car c'est bien de cela qu'il semble s'agir. L'origine, en l'occurrence effondrée, exige en tant que telle une "construction prodigieuse» (wundervollerAufbau) comme il est dit en «langue fondamentale» (Grundsprach). La crise que racontent les Mémoires et d'où la geste s'engendre n'est pas à l'origine, mais crise de l'origine, absence d'une coupure inscrite - ce qu'est l'ombilic - qui ordonne au sujet ce «tricotage d'oppositions " 8 constituant sa réponse à l'Autre. Les voix s'imposent à Schreber en un parler de nerfs ou «langue de fond" pour l' "entretenir du processus en cause» (13), autant dire pour l'informer en même temps que pour l'éprouver, vérifiant sans relâche la vivacité de sa pensée dans le but de confirmer le caractère incurable de sa maladie nerveuse pour enfin le «laisser en plan» et l'abandonner à la putréfaction (61). Il s'agit, on le devine, dans le "complot" tramé d'abord entre Flechsig et Dieu, d'accomplir jusqu'au bout le meurtre d'âme non achevé tant que DanielPaul, héritier direct de cette cassure et lié intimement à l'héritier Flechsig, résiste par un exercice perpétuel de la pensée qui tend à prouver qu'il n'est ni mort physiquement ni mort psychiquement, c'est-à-dire ni idiot ni imbécile ni fou.
Penser s'impose donc comme la réponse obligée au surgissement des voix qui racontent du même coup - et donc posent - l'origine comme cause. Dans la nécessité contraignante qui saisit Schreber après l'effondrement, dressé au centre d'un afflux de rayons, on voit bien la dimension suturante que constitue pour lui son délire qui commence précisément en tentant de faire origine. Car il n'y a pas d'autre origine que celle qui ici se constitue du délire même, ce dernier - Schreber est fort précis là-dessus commençant avec l'imposition de la fameuse phrase qui compose le noyau de la maladie, cette idée que tout de même ce doit être une chose singulièrement belle que d'être une femme en train de subir l'accouplement ${ }^{9}$.

Cette féminité «en acte» - et en procès, on le verra de même que la figure du Juif éternel (53) qui y est inextricablement rattachée puisque la transformation en femme - ou éviration - et le renouveau de l'espèce incombent à celui qui soutient seul sa propre causalité, deviennent dès lors les points d'attache de l'ombilic ${ }^{10}$. La femme et le Juif éternel (Der ewige Jude) sont certainement, dans nos cultures, les figures primordiales du refoulement. On notera d'ailleurs que le versant «inférieur» du Dieu schreberien est sémitique et féminin ${ }^{11}$. Cette double place tenue et gardée par Schreber en tant qu'elle est celle-là même de sa rédemption constitue la réponse forgée à l'appel de l'Autre (appel venu de et lancé à l'Autre) ${ }^{12}$. Le féminin, on le sait depuis Freud, est ce qui infère la castration et fonde le refoulement en lieu et place de l'origine; le refoulement, comme effet constitutif de la «révélation" du manque dans l'Autre - autant dire de sa jouissance -, fait l'origine, la pose en tant que nécessairement barrée d'un signifiant qui la situe au-delà. Le refoulement originaire - refoulement d'un signifiant (conglomérat de matières verbales) consubstantiel au voisinage et au corps qui en subit l'effraction, inscrivant à cette place ouverte dans la surface psychique, pour reprendre la métaphore freudienne, quelque chose, un représentant de l'irreprésentable, plutôt que rien -; ce refoulement, donc, est originaire dans l'unique mesure où il permet de postuler l'origine... perdue. 
Quant au Juif, il est en quelque sorte l'inventeur du Livre qui raconte et fonde cet effet logique de la parole comme Loi. L'invention juive d'une transcendance nommée "Père», faisant Loi et générant l'interdit de représentation, met en scène et en histoire ce refoulement premier, condition de la «rédemption» du sujet comme sujet parlant. À défaut de quoi, celui-ci n'a plus qu'à se vouer corps et biens au gouffre qui s'ouvre et l'emporte, contraint qu'il est d'être sujet à la parole de l'Autre.

\section{LE PARLER EST UNE ABONDANCE D'ABONDANCES}

Si la folie de Schreber nous renseigne sur la perfidie d'un Dieu en mal d'ombilic, cherchant à s'arracher au pouvoir d'attraction de celui qui ne peut embrayer son Nom qu'à partir des rayonscordons insécables, la cabale entrevoit quant à elle, si l'on peut dire, la folie d'un rapport à Dieu qui ne tiendrait pas compte de l'éclipse de l'Autre, au commencement. Dans cette optique, elle impose au cabaliste la prise en compte d'un manque de Dieu à l'origine, d'un retrait ou d'une disparition du divin contracté (Tsimtsoum) dans les 22 lettres de l'alphabet hébraïque ${ }^{13}$. Le pouvoir de ces lettres exige donc du cabaliste la prise en compte du secret qu'elles recèlent et de l'étude qu'elles appellent. L'Épître des sept voies d'Abraham Aboulafia (1240-1291) nous rappelle à quelque chose qui permet peut-être, par l'élaboration analytique qu'il déploie, de saisir la dimension éminemment universelle d'une structure propre au sujet parlant, et que Schreber, quant à lui, expose au dehors au point de n'en pouvoir disposer que par le biais du délire.

Cette école extatique soutient en effet que le rapport entre le monde d'en haut et le monde d'en bas ne passe pas - comme pour le Logos grec - par le principe d'une médiation, émanation, mais par celui du dibbour (parler) qui est conjonction, parole de conjonction. Aboulafia défend la thèse d'un Logos hébraïque, principe de cohérence de la pensée juive, principe de mobilité dont le savoir prophétique serait le mode de connaissance. On comprend que le terme Logos, qui dit bien l'enjeu intellectuel convoqué, désigne toutefois des spécificités hébraïques qui n'ont plus guère de liens avec la notion grecque.

Le parler est une abondance d'abondances (chefa chofea) Iqui vient] du Nom, au moyen de lintellect agent [pour s'appliquer] sur la faculté rationnelle, comme dit le Maitre. (Aboulafia, 1985: 19)

Sans vouloir développer ici les complexités de cette cabale philosophico-prophétique d'Aboulafia, on peut en tout cas souligner au passage le rapport qu'elle entretient avec la langue et la lettre, et rappeler, comme le fait Marc-Alain Ouaknin (1992: 9), que "cette approche du judaïsme a tellement été occultée et refoulée qu'elle apparaît aux yeux des spécialistes comme un délire ou une hérésie». Chaque fois qu'on approche au plus près de cette vérité d'un lien physique, corporel, au Verbe, il semble, en effet, qu'on s'approche du même coup de la folie. L'important pour nous est que la langue hébraïque constitue pour la conscience hébraïque un réel immédiat, la matière même du monde, autant dire sa "nature». Cette conscience particulière, que la cabale cultive jusqu'en ses plus baroques dépliements, sait d'une certitude absolue que toute réalité, la plus physique, la plus dense comme la plus lourde, est d'abord réalité de langue et de mots. «La vibration infinie de la voix porte l'univers, et [...] les mots qui la modulent en forment les structures et les figures» (Aboulafia, 1985:19). Ce réel premier, toute la tradition juive s'en réclame pour soutenir la rigueur intellectuelle de ses méthodes d'interprétation du texte. Mais le lien extatique à la manifestation dans le monde des 22 lettres de l'alphabet appartient à une branche de la cabale qui permet d'éclairer d'une manière nouvelle le réel schreberien. «Au début est le parler (dibbour), écrit Aboulafia, et le début de la conjonction (hibbour)" (ibid.). Conjonction qui veut dire aussi compagnonnage, et que la métaphore de l'«embryonnement» et du nombril (ibbour) met en rapport direct avec ce qui sépare l'embryon de la mère et ce qui unit les séparés dans l'acte de conception.

La contraction ou séparation originelle met en place après la création un espace d'entre-deux entre créature et créateur, un vide, une disparition d'où va 
jaillir l'abondance du parler comme trace, fil, afflux, rayonnage de lumière. La lettre occupe pour ce philosophe contemporain de Dante la place de l'ombilic; trace de l'union et de la séparation qui rappelle le vide, manque ou trou à l'origine de la création, et qui rassemble le sage et l'ignorant, tout être de parole autour de la lettre comme présence. Le flux d' «abondances" - ce Logos est en effet une surabondance du Nom - relie directement le Haut et le Bas sans médiation ni intermédiaire. Le rôle de l'encre devient, dans cette logique, primordial. «Il en est de l'encre comme de la liqueur séminale porteuse des formes humaines, matière de tout homme» (ibid.: 79). On entend bien que la dimension convoquée en ces termes n'est pas sans soutenir un rapport érotique à l'Autre qui travaille aussi toute la tradition juive.

Rapport érotique où la problématique d'engendrement demeure toujours à entendre sur le double plan de la création et de la pro-création.

Ce regard rapide vers la conception cabalistique du monde ouvre, il me semble, une voie de rencontre entre le délire schreberien et la tradition juive qui, audelà du simple plaisir analogique qu'elle procure, contribue à saisir la vérité universelle que ces deux «révélations» ordonnent: la matérialité de la parole est condition de la survie humaine. Véritable

Nebenmensch, environnement primordial dont la psychanalyse a exploité la consubstantialité avec le corps, cette matérialité se donne à voir au sujet psychotique et s'impose à la méditation extatique du cabaliste. Cette consubstantialité de la parole et du corps fonde, comme on sait, l'interprétation psychanalytique; elle pose de surcroît le principe du refoulement dont Freud - c'est ce qui caractérise sa lecture du cas Schreber - a reconnu l'inespérée mise au jour dans le délire du Président. La réalité psychique, dont la modalité est l'inconscient, se trouve chez le psychotique projetée au dehors en une construction merveilleuse et intensément souffrante. Elle est, pour le cabaliste, éprouvée dans sa dimension structurante, et portée au registre de la contemplation à partir d'un Logos reposant entièrement sur les pouvoirs de la lettre hébraïque. Contemplation qui, à la différence du délire, vise la prise en compte de cette réalité ombilicale comme instauration de l'éthique: Loi «naturelle» du dibbour.

\section{D'UN DIEU SUPPOSÉ NE PAS SAVOIR}

Pour la tâche que je m'étais fixée, et qui était à tout instant de convaincre un Dieu qui ignorait tout de l'être humain vivant, de l'intégrité de ma raison, la lumière nécessaire à toutes les entreprises humaines m'était devenue presque plus indispensable que le pain quotidien. (173)

Le refoulement originaire concerne, dit-on, un signifiant. Signifiant de ce défaut, béance, faille ou crise dans l'Autre, qui fait ombilic, marquant d'un autre nom que le mien la division d'où je suis issu. On ne s'étonnera pas que le défaut de ce «signifiant du défaut" livre le sujet, qui s'approche du puits en question, aux violences d'un déferlement et d'une attraction terriblement menaçantes. Le manque fait alors retour du dehors comme défection, méconnaissance, précarité, effondrement qui abandonne le sujet aux «miracles du signifiant». Car ce signifiant non refoulé, manquant à sa place, Schreber nous révèle qu'il apparaît dès lors partout sous la modalité de l'absence: phrases tronquées qui s'imposent à son esprit et le contraignent de les achever pour prouver sa vivacité; jacasseries incessantes ou propos in-signifiants qui envahissent tout l'espace sonore; euphémismes, termes signifiant le contraire de ce qu'ils disent, le mot juste étant occupé lui aussi à dire son contraire; métaphores multiples comme "retirer les bottes» pour dire éviration, ou "harnachement» pour vêtements (166); toutes formules qui, dans la conception des voix, ne cessent d'indiquer l'absence d'un signifiant forçant le sujet à le dire, le penser, le trouver sans ambiguité de sens. L'opération mentale consiste en l'occurrence à combler en permanence les brèches du discours. Lisant les Mémoires, on finit par entendre que les forces parlantes s'occupent essentiellement à mimer le défaut du signifiant, le trou qu'il laisse devenant le lieu d'épuisement du sujet voué à l'incarner pour ne pas être laissé en plan, certes, mais aussi pour sortir Dieu de la «bien précaire posture» (29) en laquelle il est tombé. 
En fait, on s'aperçoit que, dans l'Autre, une exigence se fait entendre qui n'est que l'exigence du signifiant en tant que tel. Ce que Lacan (1975: 101) a remarquablement analysé:

Le signifiant subit lui-même de profonds remaniements, qui vont

donner cet accent si particulier aux intuitions les plus signifiantes pour le sujet. La langue fondamentale du Président

Schreber est en effet le signe que subsiste à l'intérieur de ce monde imaginaire l'exigence du signifiant.

Ce que Schreber appelle la "politique» de Dieu, et qu'il reconstruit en tous les détails que la paranoïa impose, désigne très précisément cette perturbation de la relation à l'Autre où c'est d'abord au lieu de l'Autre que le sujet est "pris pour» et donc assujetti à cette méprise, forcé qu'il est d'y remédier par le travail incessant du délire. La division de Dieu est dès lors manifeste: la politique consacrée à la mise à l'épreuve de Schreber, aux violences des hallucinations, des «malices» et ignominies corporelles, contraint la pensée et le corps à des réponses trop vite rendues automatiques et du coup en proie à une rapide déperdition de sens. Réponses dictées par les voix, elles aussi inscrites dans le registre divin. Royaume de devant ou Vestibule du Ciel, Royaume de derrière, divisé en Dieu d'en haut et Dieu d'en bas; le paysage céleste du névropathe est livré à une mathématique de division qui n'est pas sans évoquer une fois encore la causalité même du «Révélé». Entre ces deux Royaumes, Schreber «tricote» littéralement son délire suturant. Les voix informent donc Schreber d'une méprise, et tout le prodige de la psychose vient en réponse à ce dire. Réponse délirante qui conserve toutefois ce noyau de vérité que soutient la révélation: il n'y a d'origine qu'au champ de l'Autre.

Ce champ de l'Autre se donne à voir comme un champ de nerfs qui produisent, par branchements plus ou moins intenses, des effets de corps redoutables. Ce qu'on apprend dès l'ouverture du livre de Schreber, c'est l'ignorance radicale de Dieu concernant les êtres vivants et leurs productions. Nulle toute-puissance ou omniprésence de Dieu, au sens où Dieu aurait été en mesure de «sonder les reins et les cœurs» soutient Schreber (20). Ce savoir serait d'ailleurs sans objet puisque Dieu ne commerce qu'avec les âmes des défunts sauf exception rare et provisoire. N'eussent été la crise dans l'ordre de l'univers et la maladie de Schreber, qui ont eu pour effet de diriger les rayons divins en ce centre d'attraction unique, Dieu n'aurait pas eu à apprendre quoi que ce soit. Et Schreber constatera à plusieurs reprises à quel point il est inéducable ${ }^{14}$.

C'est au chapitre IX que Schreber, relatant une modification dans ses échanges avec les rayons ou nerfs, nous renseigne sur le statut finalement scripturaire de l'arrimage aux terres des rayons qui relie Dieu à Schreber et aux corps célestes. On apprend en effet que si, depuis le début, il est clair pour le Président que Dieu ne connaît rien aux affaires humaines ni au vivant, qu'il est pour ainsi dire sans savoir sur l'humanité et ses exploits, un système de prise de notes - véritable dispositif divin venant au secours de son défaut de puissance - permet tout de même, in extremis, de le renseigner. Au secours d'une toute-puissance divine défaillante, les rayons convoquent des êtres déshabités par l'esprit mais qui, traversés par les rayons-nerfs, procèdent à l'enregistrement des données sur le monde dont la source unique est Schreber lui-même, sa pensée.

On tient à jour des livres ou autres écritures, dans lesquels depuis

des années déjà sont consignées toutes mes pensées, mes façons de parler, dans lesquels sont recensés tous mes objets usuels, toutes les choses qui se trouvent en ma possession ou autour de moi, ainsi que toutes mes relations. (126)

Schreber ne peut dire qui prend ces notes, mais il suppose que des êtres siégeant dans les corps célestes et apparemment dépourvus d'intelligence assurent, par branchement aux rayons rattachant le malade à Dieu, la transcription mécanique et totale de sa réalité. «Les rayons qui passent par là leur forcent pour ainsi dire la plume dans la main" (127).

Cet arrimage proprement littéral n'est pas sans produire les atteintes que l'on découvre à la lecture de ces hauts faits du malade des nerfs: atteintes à l'intégrité corporelle, à la virilité et à la raison. Car ce 
dispositif de prise de notes n'est que le second tour donné à la puissance d'attraction que constitue le sujet Schreber, second tour qui porte le combat explicitement sur la scène aimantée d'une trace. Ce n'est pas le savoir de Schreber qui est mis en cause, ni le sens de ses pensées mais leur inscription qui les situe radicalement au registre du pur trait; exorcisme qui là encore pointe dans l'Autre l'arrachement, le retrait... planifié, programmé.

Le stratagème, donc, était le suivant; dès que se manifestait en moi une pensée que j'avais déjà eue avant - pensée qui par conséquent avait déjà fait l'objet de notes prises (et un tel retour n'était-il pas inévitable pour nombre de pensées? ainsi de la pensée "maintenant je vais me laver», quand c'était le matin, ou "voilà un beau passage», quand je jouais du piano) - dès qu'on décelait donc en germe ce genre de pensées, les rayons susceptibles d'être attirés à proximité de ma personne étaient munis, en guise de viatique, d'un «nous avons déjà» (prononcé "zavons déjà»), sous-entendu: "noté cela», formule qui les immunisait [...] contre la force d'attraction en question. (132)

L'ordre divin, on l'a compris, est perturbé par l'arrimage des rayons au corps de Schreber; arrimage qui est une conséquence de la crise de l'origine dont le débranchement, comploté par les âmes, laisserait cet homme pour mort. En attendant, nous assistons à ce qu'il faut bien appeler les miracles du signifiant, effet de la toute-puissance entamée, «louche» et «mauvaise» de Dieu à l'endroit du Président. Les rayons, Schreber nous l'explique en long et en large, sont en quelque sorte des «fréquences vibratoires correspondant aux mots qui les composent» (142), mots ressentis directement comme une douleur d'arrachement inscrivant dans le corps du malade les efforts des rayons pour se dégager de son emprise d'attraction. Le chapitre XI des Mémoires est entièrement consacré à cette entame perpétuellement perpétrée sur le corps dès lors «morcelé». Travail incessant de destruction et de restauration, dû à la disparité des rayons partagés entre les purs et les impurs; les premiers entièrement voués à la création devant restituer ce que les seconds - espiègles, galopins et malicieux (149) - ont ravagé. L'éviration est bien sûr au centre de cette incessante métamorphose qui ne laisse cependant aucune partie intacte: poils de barbe, de moustache, stature corporelle tout entière soumise à un tassement osseux, cœur, poumons, larynx, estomac, système immunitaire, cordon spermatique, pourriture du basventre, tête, yeux, paupières, rien n'échappe aux assauts violents des «rayons traceurs" (155) qui font de Schreber un véritable complexe d'inscriptions et de ratures.

On pourra se représenter à quel point tous ces processus pouvaient faire naître de sensations pénibles lorsqu'on se souviendra qu'autour d'une tête unique rôdaient les rayons d'un univers entier [...] qui s'efforçaient comme pour un écartèlement de la disloquer ou de la faire sauter. (155)

Telle est la Politik der Halbheit (politique de demimesure, de semi-turpitude et d'irrésolution) particulière à ce Dieu schreberien (56); politique de la lettre, pourrait-on avancer, dans la mesure où c'est le corps même du sujet psychotique qui se fait à la fois le site du retrait (contraction et arrachement) et du trait signifiant (ombilic littéral).

\section{LIBÉRER LES ÉTINCELLES DE L'ÂME}

Le lien étroit entre Schreber et Dieu n'est certes pas facile à dégager. Si l'on parvient, comme lui et avec lui, à reconstruire les différentes forces divines à l'œuvre dans ce rapport étroit du malade à l'Autre, on a envie de donner raison à Freud (1995: 27) lorsqu'il affirme qu' «il est besoin d'une bonne dose de confiance pour persister à attendre que dans ce “délire” on puisse trouver de la méthode». La méthode est une chose, la cohérence une autre. Il se trouve qu'à traverser les Mémoires d'un bout à l'autre, la fatigue du voyage se voit compensée par l'impression d'une grande cohérence quant à la teneur de ce lien à Dieu. Teneur érotique, il va sans dire, que la béatitude de Schreber établit comme un devoir rendu à l'ordre universel, et qui lui permet ultimement de soutenir sa métamorphose en femme comme l'accomplissement d'un futur à la fois certain et infiniment reporté. 
La cabale soutient de son côté l'exigence éthique d'un rapport érotique à Dieu qu'elle dispose, dans la logique du Tsimtsoum, comme un impératif donné à l'homme, après le retrait de Dieu et son explosion dans les 22 lettres de l'alphabet hébraïque - explosion dont les flux d'abondances sont livrés, rappelons-le, à la contemplation et à l'étude humaine -, impératif de libérer les étincelles divines contenues dans ces flux et de les rendre à Dieu. Le cabaliste affirme ainsi que l'éthique consiste à soutenir Dieu, autant dire à l'aider à recouvrer sa perfection perdue et, avec elle, à parachever le monde de la création. Cette logique de l'arrachement, du déferlement, de l'attraction puis de l'union réciproque, que l'on découvre dans la troublante épopée schreberienne, trouve donc une résonance dans la mystique hassidique, par exemple, où l'étude de la lettre relève de sa brisure, explosion rejouée sur tous les matériaux que sont les consonnes et les points-voyelles entourant la lettre proprement dite, au profit d'une libération du désir perçu comme condition de la perfectibilité du monde, de son accomplissement... à venir.

L'éthique est ainsi brisure, cassure, fissure. Elle est d'abord éclatement du mot en ses lettres, soulignant que le rapport entre les voyelles et les consonnes n'est pas une évidence et qu'il faut y introduire le Désir. (Ouaknin, 1992: 73)

D'autre part, on peut dire que la composition subdivisée du Ciel schreberien rappelle sur le mode caricatural l'arbre séfirotique qui donne aussi à Dieu un "corps» constitué de ramifications rayonnantes. Si Schreber n'est manifestement pas un sujet mystique dans la mesure où son délire ne construit pas une méthode de rencontre, mais compose la description positive et «scientifique» d'un Dieu qui ne concerne que lui, ce Dieu n'a tout de même pas d'autre sens que de rendre intelligible la dimension désirante et «attractive» du signifiant et de la parole.

En fait, l'analogie entre le délire de Schreber et la cabale n'est pas si étonnante si l'on comprend que le cabaliste, comme le malade des nerfs, projette l'intégralité de son être et de son essence dans l'infini. À la différence du malade, pourtant - et parfois au risque de le devenir (Wolf, 1998) -, ce principe de projection est une méthode qui fait l'objet d'une transmission et d'un enseignement.

Lorsque le cabaliste présuppose l'existence d'innombrables étapes intermédiaires entre la nature limitée de l'homme et l'infini, il se figure des parois spirituelles qui se feraient face, ou des surfaces de projection parcourues par des rayons émanés de l'infini et dirigés vers l'homme. (Langer, 1990: 36)

La luminosité de ces rayons solaires varie selon leur proximité et leur éloignement et de ce fait se décompose en différents éléments primordiaux avant de parvenir à l'homme. Les innombrables étincelles du Désir se libèrent et donnent naissance aux mondes supérieurs. C'est là que s'établissent les séfirot (de safar, compter), c'est-à-dire les dix puissances divines ou Noms divins (Sagesse, Charité, Jugement, Intelligence, etc.), les "palais merveilleux» et les âmes désincarnées dont traite la cabale. Cette méthode de projection n'est pas sans mettre en lumière ce que le délire de Schreber quant à lui actualise dans une expérience frontale: de part et d'autre il s'agit d'une rencontre avec l'origine comme cause... à restaurer.

TROU, NERFS, RAYONS: ATTRACTION ET DÉFERLEMENT

C'est contre leur gré et dans une sorte d'effroi que les rayons, qui étaient habitués à une impassibilité sacrée comparable à celle qui règne ordinairement sur les plus hauts sommets montagneux de la terre, doivent désormais prendre leur part de toutes mes sensations auditives [...]. (83-84)

Si la fonction symbolique propre à l'instauration du signifiant du manque dans l'Autre est nécessaire à soutenir l'imaginaire du corps comme Un, on comprend que la déstructuration corporelle que subit Schreber relève, comme il le dit, de la faille qu'il sait béante dans l'ordre de l'univers. Le système complexe des rayons tendus en réseaux apparait en effet suspendu au-dessus de cette brèche, le branchement aux nerfs de Schreber constituant justement la conséquence première et dernière de cette déchirure apparemment irrémédiable. Dans l'ordre du délire, le trou que le «meurtre d'âme» a engendré, et les 
déviations conséquentes opérées par les âmes examinées (purifiées) s'arrogeant des rayons divins pour un mésusage dont Schreber est la cible, agit comme un déferlement qui emporte «les rayons d'un univers entier " à ce lieu unique d'attraction.

Il est assuré [...] que Dieu qui auparavant séjournait dans un éloignement inoui de la terre, a été obligé de s'approcher plus près, et de là vient que la terre est devenue le théâtre immédiat et continuel de miracles divins [...]. Ces miracles convergent avant tout sur ma personne et à mon voisinage immédiat. (87)

Cette dérivation de l'origine que la puissance d'attraction des nerfs du malade conduit constitue l'essentiel de la geste qui, on l'entend de plus en plus, met en danger sur un axe de réciprocité et Dieu et le sujet «branché». Si le système de prise de notes n'est encore qu'un stratagème pour inhiber l'afflux des rayons afin de les dégager promptement de la force d'attraction (139), cette dernière, qui constitue précisément la maladie de Schreber, est aussi sa seule arme contre l'abandon aux déferlements et à l'écroulement radical.

Il faudrait souligner mieux, je crois, les incertitudes que l'auteur ne cesse de disposer dans son récit; incertitudes liées au statut même de l'expérience vécue comme accumulation ET dissémination, attraction centrée ET déferlement, l'effondrement emportant, on l'a vu, les bords du trou-puits et l'univers entier à sa suite. Ainsi, le meurtre d'âme, fort détaillé sur le plan anecdotique et généalogique, se présente tout de même comme une spéculation, hypothèses et avancées venant au-devant d'une inconnue considérée comme inconnaissable. Le texte témoignage de Schreber ne livre en fait qu'un échantillon - superbement organisé - d'une expérience autrement prolongée dans le temps, en évolution constante parce que soumise à une dialectique permanente qui ne laisse au malade aucun répit. La spéculation une fois orchestrée, le doute n'opère toutefois que sur le mode rhétorique, ne touchant d'ailleurs que la signification dernière de toutes ces manœuvres; la certitude du sujet quant à sa place de destinataire du sens demeurant jusqu'à la fin inattaquable.
La dérivation - attraction et capture - déclenche donc le commencement d'une histoire mémorable dont le terme se donne de plus en plus clairement comme «jouissance de Schreber», qui est à la fois béatitude du corps et du Nom. Il est clair dès le départ que l'attraction est d'ordre érotique. Comme le souligne Freud (1995: 76):

Les rayons divins schreberiens composés par condensation de rayons solaires, de fibres nerveuses et de spermatozoïdes, ne sont à vrai dire rien d'autre que les investissements libidinaux présentés comme choses concrètes et projetés vers l'extérieur, et ils confèrent à son délire une concordance frappante avec notre théorie.

Schreber lui-même rend compte de cette fonction de volupté de manière fort précise.
L'attraction perdait toutefois ses effets effrayants pour les nerfs en question, au moment et dans la mesure où, en faisant leur entrée dans mon corps, ils rencontraient ce sentiment de volupté
d'âme auquel ils prenaient part de leur côté. Ils retrouvaient alors dans mon corps, à la place de la béatitude céleste qui s'était perdue, consistant sans doute, elle aussi, en une jouissance d'un mode voluptueux, un substitut de valeur soit tout à fait égale, soit du moins approximative. (179)

Claude Rabant a, pour sa part, bien dégagé dans l'intrication ordonnée des rayons le sens du meurtre d'âme reposant sur l'insupportable doublure de la lignée.

L'insupportable c'est qu'il y ait Schreber et Flechsig, et partant deux lignées susceptibles de se mêler, comme l'atteste l'apparition répétée d'un prénom Schreber dans la lignée Flechsig. Dualité aussi insupportable que l'existence de ces deux soleils qui surgissent dans le ciel. (1978: 185)

Dualité que l'on retrouve tout au long du délire et qui organise la cosmologie schreberienne dans son ensemble. Il s'agit apparemment pour Schreber de reconstruire le un à partir du deux. Dans la mesure où la béance dans l'Autre n'a pu se suturer d'un barrage signifiant qui signerait la place du sujet expulsé du désir béant et viendrait l'inscrire dans une généalogie - la différence sexuelle qu'implique toute naissance se 
trouvant signifiée par ce représentant du désir-, le délire vient au secours du sujet en proie au déferlement que le vide laissé ouvert entre les deux sexes déclenche. L'histoire du meurtre d'âme et de sa conséquence en une convergence des rayons divins sur Schreber - élection qui réunit toutes les dualités cosmiques et théologiques - tente de résoudre cette absence du Un sur le mode de l'Unique. L'attraction est donc l'invention d'une "physique" constitutive d'Un corps pour la jouissance.

Suivant cette logique de contrainte, le discours des voix subit une évolution constante. L'âme, Schreber nous l'a décrite dans sa fonction psychique et corporelle, c'est l'identité, mémoire et sensations. Dans le déplacement qui s'opère en Schreber et qui va de l'idée terrible de l'éviration à ce qu'il appelle sa transformation en femme par la présence des nerfs de la volupté, féminisation réalisée dans une jouissance qui concerne tout le corps, l'atteinte au sexe se trouvant reportée dans l'éternité et par conséquent rendue secondaire devant l'«évidence» de l'image d'une femme que projette Schreber (281); suivant cette évolution, donc, le principe d'attraction subit lui aussi une réinterprétation de sa causalité. En fait, le récit nous apprend qu'à travers les ignominies et les iniquités auxquelles les rayons soumettent Schreber, la question même de la causalité qui chaque fois s'impose à lui fait accéder le malade des nerfs aux lumières éblouissantes des choses surnaturelles. Stimulation intellectuelle et connaissances auxquelles le sujet ne renoncerait pour rien au monde. Voici la situation:

Ces conjonctions sans suite qui faisaient intrusion dans mes nerfs et qui exprimaient les liens de causalité ou tout autre relation circonstancielle: «Pourquoi parce que», "Pourquoi parce que je», "si ce n'est que», "au moins», m'obligeaient littéralement à réfléchir sur quantité de choses à côté desquelles les gens passent négligemment, et contribuaient par là même à un approfondissement de ma pensée. (228)

Dans ce travail imposé sur la causalité, la question du nom apparaît centrale. La cause du nom, en effet, s'impose à l'esprit de Schreber articulée à «toute une série de démarches de recherche sur les fondements et l'origine des noms de personne». Si la réponse obligée à la question «Pourquoi cet homme s'appelle-t-il Schneider?» est «Parce que son père lui aussi s'appelle Schneider", aucun apaisement n'en résulte et le malade se voit confronté à l'impropriété du nom, à son inadéquation par rapport à l'identité, donnant en cela même à la Denkzwang (contrainte à penser) sa figure éminente. Schneider (tailleur, coupeur) mis pour Schreber ne permet pas d'annuler l'enjeu qui se signale ici. La question de la cause du nom dit bien que ce qui rend le nom transmissible c'est un point zéro, lieu de bifurcation où la vie et la mort se séparent, où l'identique et le non-identique se conjoignent pour représenter une jouissance fondée sur la division, point zéro où la cause s'annule, où le père est dessaisi de sa puissance pour fonder la métaphore de cette bifurcation comme dessaisissement et transmission. Ce qui contraint Schreber à la pensée insatiable sur les fondements et l'origine du Nom c'est son impossibilité d'accéder à ce dessaisissement fondateur du sujet de la parole. À la place de la bifurcation, il n'éprouve que de l'insupportable fraction dont la cause est forclose, livrant les rayons-signifiants à la souffrance d'un Dieu qui ne tolère pas la perte. D'où, écrit Claude Rabant (1978: 183), leurs appels au secours. «D'où l'exigence d'un Retour, qui fonde l'ordre apparent de l'univers sur un cercle, ou plutôt une ellipse éternelle». L'attraction n'est pas autre chose que ce retour au foyer où Schreber se dresse, annulant la division par un principe de fusion et d'accumulation dont la jouissance est toute féminine. Le délirant se constitue en l'Unique foyer d'attraction du sens dont la «J'ouïe sens» - pour reprendre le jeu de mots de Lacan - est déterminante de sa rédemption et de son élection comme ombilic du monde.

\section{UNE CHOSE SINGULIĖREMENT BELLE}

Ce qui est exigé, c'est que je me regarde moi-même comme homme et femme en une seule personne, consommant le coït avec moi-même. [...] Dieu exige un état constant de jouissance, comme étant en harmonie avec les conditions d'existence imposées aux âmes par l'ordre de l'univers. (282) 
Le chapitre XIII des Mémoires se consacre à la description de «la volupté d'âme comme agent de l'attraction et de ses conséquences». Le récit porte sur un tournant de la vie du Président, daté du mois de novembre 1895. Il s'agit, pour être précis, du moment où l'éviration, considérée comme un impératif absolu pour la fécondation par les rayons divins en vue de l'engendrement d'une nouvelle race d'hommes, perd de son caractère humiliant du fait que Schreber est convaincu qu'aucun être vivant ne pourra y assister puisqu'il est effectivement le dernier homme. Le tournant marque donc le moment où le Président accepte de se consacrer entièrement à la féminité, culte et volupté constituant le service rendu aux rayons et à Dieu lui-même. Après cette date et le retour de Schreber à la réalité des vivants, l'humiliation d'une métamorphose en femme au regard des autres hommes est résolue par l'idée que cette métamorphose se produit à une lenteur telle qu'aucun vivant actuel ne pourra y assister. L'éternité est ici le vecteur du salut. Schreber accepte donc enfin et en pleine conscience d'«inscrire sur ses étendards le culte de la féminité».

Mais ce chapitre est certainement le plus éclairant quant à la dialectique de fractionnement qui s'opère en Schreber. La dualité de Dieu est en quelque sorte, à cette date de l'automne 1895 , ce qui s'impose au malade comme le commencement d'une lutte de cinq ans entre l'inférieur et le supérieur, le féminin et le masculin, bref entre Ariman et Ormuzd jusqu'au triomphe du premier qui rend inopérante la politique de destruction convertie en taquineries inoffensives. Ce qu'il faut entendre, somme toute, c'est que l'exigence de béatitude qui s'impose à Schreber à cette date comme condition de sa guérison et de la rédemption du monde, les deux se trouvant - malheureusement - confondues, instaure un duel entre le féminin et le masculin explicitement éprouvé comme facteur de renforcement de l'attraction propre aux nerfs de Schreber. La jouissance ou béatitude, on l'entend dans l'histoire de cette dualité matée, met en cause, non seulement la toute-puissance de Dieu - qui depuis le début est empêchée et même révélée inexistante -, mais l'incapacité de Dieu à apprendre. L'accession à l'état de béatitude constant relève donc d'une complicité entre le Dieu inférieur et Schreber pour démobiliser l'impression d'imbécillité qui risque de permettre le retrait de rayons et de laisser le sujet en plan. Le Dieu de jouissance se révèle donc aussi un Dieu au service de la pensée contraignante qui vient en réponse aux pièges et perfidies du Dieu supérieur.

Dieu est donc duel. On s'égare, par ailleurs, dans la lecture des Mémoires qui racontent le fractionnement incessant des âmes examinées en rayons, les réseaux de "vibrations parlantes" démultipliant cette dualité à l'infini d'une circulation devant aboutir, par passage en un foyer exorbité (lieu des prises de notes, on l'a vu) au foyer principal incarné par Schreber. À cette construction prodigieuse et si difficile à percevoir dans le dédale historicisé de la geste délirante correspond sans aucun doute l'institution de la Beauté.

Cette «chose singulièrement belle" qui s'impose à l'esprit de Schreber au commencement de sa maladie et qui, on le voit, constitue le noyau de la métaphore délirante offerte à Dieu comme «l'image d'une femme plongée dans le ravissement de la volupté»(281), noue le délire à une condition esthétique qu'il semble important de rappeler. De quoi s'agit-il au juste? D'ordre, il va sans dire; de la restauration d'une harmonie perdue que la "chose singulièrement belle" (eigentlich recht schön) réinscrit au cœur de l'histoire: future d'une éviration à portée messianique. On se rappellera à ce titre la délirante théorie de Wilhelm Fliess dont Freud - malgré l'aveuglement propre au transfert qui caractérise à cette époque son lien à l'ami - avait très tôt décelé le caractère essentiellement harmonieux, la pensée de Fliess se trouvant sollicitée par la primauté d'une cohérence formelle:

Je commence à saisir ton point de vue, écrit Freud: tu recherches non point ce qui présente un intérêt culturel, historique, mais la beauté absolue, dans une harmonie de forme et d'idée et dans les sensations essentiellement plaisantes de couleur et d'espace. 15

La beauté est équivalente à la conformité à l'ordre de l'univers, conformité dont Schreber se dit quant à 
lui responsable. La beauté est donc, en ce sens, travail d'accomplissement dont le repérage éminemment esthétique apparaît dans les mises en scène au miroir qui forment l'aboutissement de la maladie au moment où l'auteur entreprend d'écrire son livre. Le livre, sa composition - chaque lecteur le perçoit à la lecture est directement soumise à ce devoir de beauté absolue qui constitue, pourrait-on dire, la part la plus visible de son appartenance au genre "pathologique».

Si Octave Mannoni a bien montré que la rigueur ici à l'œuvre n'était pas celle de l'écrivain, il reste à dire comment la jouissance féminine, perçue par Schreber comme la plus belle et la plus juste image de l'accomplissement du monde en cette volupté «pieuse» et sacrée (285) à laquelle il accède, coïncide avec la composition d'un livre qui s'écrit comme l'instrument de la preuve rationnelle et universelle de cette jouissance vraie.

Il n'est absolument pas dans mes intentions de faire de la propagande en faveur de mes croyances personnelles aux miracles et en faveur de mes thèses sur les choses divines; je veux bien plutôt me borner à coucher sur le papier ce que j'ai vécu et ce que j'ai expérimenté, fermement confiant qu'un tableau d'ensemble des phénomènes prodigieux qui peuvent être constatés sur ma personne, et qui ne pourront vraisemblablement à l'avenir que se manifester de plus en plus clairement, forcera de lui-même la voie à la reconnaissance de la vérité pour tous - dût-il s'écouler encore d'ici là bien des années. (242)

La beauté n'a d'égale que la rationalité qui la soutient et qui noue de manière troublante le délire à la théorie. S'il y a proximité de structure entre le délire et la théorie - comme constructions interprétatives du monde et des phénomènes de l'expérience -, la distinction entre les deux demeure primordiale pour soutenir la pensée d'une éthique et d'une transmission.

On peut sans doute se demander, au bout de ce parcours, quel est ce savoir du délire que la théorie n'est pas sans reconnaître comme sien et que la mystique élève elle aussi au statut de vérité universelle. Car il y a bien un savoir au noyau du délire, savoir dont nous ne pouvons faire l'économie au nom de la Raison qui en serait l'exclusif dépositaire.
EN TOUTE RATIONALITÉ

Désormais, indubitablement, j'avais pris conscience de ce que

l'éviration était, que cela me plaise ou non, un impératif absolu de l'ordre de l'univers et que, en toute rationalité [aus

Vernunftgründen], il ne me restait plus qu'à me familiariser avec l'idée d'être transformé en femme. (177)

C'est donc travesti en femme et passant sa journée devant le miroir que le Président Schreber entreprend d'écrire son livre. Il importe à présent de dire comment ce livre, qui participe intimement du délire, met en ouvre aussi un dispositif particulier où la place du lecteur - sa construction comme intersubjectivité - n'est pas indifférente. La lutte de Schreber avec Dieu a pour but de sauver sa raison, mais ce salut va de pair avec une prise en charge personnelle de l'impératif absolu.

Le débat de Schreber [...] est un débat des Lumières: une hutte, non seulement pour sauver sa propre raison [Verstand], intelligence, entendement, dont il ne cesse d'affirmer, de bout en bout, la certitude, mais pour trouver une assise à la Raison [Vernunft], pour trouver en elle un Grund, un fond, c'està-dire une limite où l'entendement prenne appui. (Rabant, 1978:185)

Ce Grund, le lecteur en devient la garantie dans la mesure où il est interpellé précisément à cette place que la demande de reconnaissance dispose, demande que Schreber formule comme une plaidoirie où le lecteur est convoqué en tant que juge et disciple. «Dans l'éventualité, que je considère comme probable, où mes Mémoires - à savoir le présent travail - devraient un jour devenir une source importante de connaissances pour l'édification d'un système religieux tout à fait neuf» (187), écrit Schreber; ce qui dit bien à quel point sa demande repose sur la certitude qu'il demeure, lui, directement branché sur le divin, «le parti le plus clairvoyant et du même coup le meilleur» (188). L'impératif absolu fait ici fonction de Loi, mais à la différence de la Loi éthique, elle ne semble concerner que Schreber et s'éteindra avec lui, le lecteur devenant le dépositaire du témoignage pour la postérité.

Ce qui, par contre, concerne le reste de l'humanité, c'est le savoir qui se dégage des articulations du délire. 
Savoir qui vise directement et exclusivement le signifiant en tant qu'il est motivation du sens et du désir. Le délire, en effet, met en scène un «arrimage» qui, s'il est pour Schreber tout physique, incarne justement la notion freudienne de représentant psychique comme condition d'existence du sujet de la parole. Le réseau de signifiants - rayons vibratoires de mots, dit Schreber - qui lie le sujet à l'Autre suivant la loi d'attraction propre au refoulement, permet à cette liaison d'opérer dans le registre symbolique, c'est-à-dire dans le registre d'une certitude du sens dont le réel est assuré au champ de l'Autre par le barrage du refoulement qui m'interdit, justement, d'avoir à répondre de cette certitude. Le refoulement apparait donc comme étant ce qui pose l'origine au-delà du «je» qui ne peut, lui, que commencer dans l'effet de cette coupure. L'assujettissement au symbole qu'est ce signifiant consubstantiel au corps de sensations - plaisir, douleur, jouissance - qui en est la surface d'inscription mémorielle interdit toute assomption de l'origine. Pour Schreber, cette assomption catastrophique non barrée livre le corps de sensations (voir, entendre, éprouver) aux assauts déferlants du signifiant, mais le travail acharné et interminable du délire a pour avantage de révéler au monde la matérialité oubliée du langage.

Au commencement, dit la cabale, il y a le parler et le parler passe par la lettre.

Étant donné que les lettres sont composées de matière et de forme, et que les mots sont composés de lettres, et que les choses sont composées de mots, il est nécessaire que les lettres nous renseignent sur le réel tout entier. (Aboulafia, 1985: 82)

Ainsi s'impose à quiconque s'avance vers l'origine de toute chose cette lettre-corps qu'est l'ombilic. Ombilic de la voix qui depuis toujours m'arrive et me parvient du lieu de l'Autre.

\footnotetext{
* Cet article fait partie de travaux réalisés dans le cadre de l'équipe de recherche L'Imaginaire de la fin, subventionnée par le FCAR.
}

\section{N O TES}

1. D. P. Schreber, Mémoires d'un néuropathe, Paris, Seuil, coll. «Points", 1975, p. 58. Désormais, je placerai entre parenthèses, à la suite des passages tirés de l'œuvre de Schreber, le numéro de la page correspondant à la première édition allemande, indiqué en marge et entre crochets dans cette édition française.

2. G. Langer, 1990: 166. Il n'existe pas de règle officielle de transcription des mots hébreux en français, ce qui explique qu'un même mot apparaisse avec une orthographe différente suivant les auteurs. Pour ma part, j'ai suivi l'usage du Dictionnaire encyclopédique du judaisme, paru en 1993 chez Cerf/Laffont.

3. Voir le remarquable développement de Rachi dont je ne retiens que ce passage: «Vraiment ce texte nous dit: Explique-moi. C'est comme nos maitres l'ont expliqué: le monde a été créé pour la Torah que l'Écriture appelle le commencement de Sa voie (Prov., 8, 22) et pour Israël que l'Écriture appelle le commencement de Sa moisson " (Jér., 2, 3). La Torah avec commentaires de Rachi et notes explicatives (bilingue), vol. 1, Genèse, Paris, Association S. et O. Lévy, $7^{\text {e éd., }}$ 1993, p. 3.

4. A. Chouraqui, La Bible. Entête, Paris, J.C. Lattès, 1992, p. 17 : «Beréshit employé substantivement n'apparaît que dans ce texte. C'est un hapax, un mot "employé une seule fois" [...]. Les rédacteurs ont voulu ouvrir la Bible par un mot inouï et unique, dont plus de deux millénaires de méditations n'ont pas épuisé le sens. En archè traduisent les LXX [Septantes], plus tard corrigés par Aquila: En kephalaïo, Entête. Jérôme, entre deux traductions grecques, hésite visiblement et choisit la solution intermédiaire In principio [...]. Par la suite, les traducteurs aggravent l'écart entre le texte et sa traduction en interprétant In principio par Au commencement, In the beginning, effaçant ainsi les significations propres du premier mot de la Bible où il ne figure qu'une seule fois".

5. J'ai travaillé avec l'édition Ullstein Buch, Frankfurt/M - Berlin -

Wien, 1973, qui reproduit aussi entre crochets les pages correspondant à l'édition originale.

6. Ainsi que nous l'indiquent les traducteurs P. Duquenne et N. Sels à l'ouverture de l'édition française des Mémoires d'un névropathe.

7. Sur le rapport que Schreber a entretenu avec la foi, on pourra lire les pages 63 et $64:$ : [...] je n'ai (depuis le temps de mon adolescence) jamais été un vrai croyant au sens de notre religion positive. Mais à aucun moment non plus je n'ai été un contempteur de la religion». 8. J'emprunte ce terme de «tricotage " à C. Melman, proposé dans son séminaire de 1994-1995 (1999: 16).

9. En allemand la phrase se lit ainsi: «Es war die Vorstellung, daß es doch eigentlich recht schön sein müsse, ein Weib zu sein, das dem Beischlaf unterliege».

10. «Éviration» est le terme choisi par les traducteurs français pour Entmannung signifiant habituellement "émasculation", "castration". Éviration est, en effet, un terme plus juste pour désigner l'opération des rayons sur le corps de Schreber; opération qui ne consiste pas en une émasculation mais accomplit plutôt une métamorphose progressive des organes sexuels, le membre viril subissant un rétrécissement, et tout l'appareil génital se trouvant absorbé dans le corps au profit d'un sexe féminin.

11. Le "Ciel» de Schreber est constitué de multiples doublures: Royaumes de devant ou Vestibules du Ciel et Royaume de derrière, luimême divisé en Dieu d'en haut (Ormuzd): Dieu éloigné, aryen et masculin, capable de restaurer la virilité; et Dieu d'en bas (Ariman): Dieu proche, sémitique et féminin, produisant le miracle d'éviration. 
12. Freud a bien montré que ce fantasme de rédemption ne concerne guère le reste de l'humanité mais "consiste essentiellement en ce qu'aucun défunt ne peut atteindre la béatitude aussi longtemps que Schreber absorbe par sa force d'attraction la masse principale des rayons divins" ; Freud, 1995: 27.

13. La notion de Tsimtsoum (contraction) - mot hébreu qui vient du verbe letsamtsem qui signifie contracter, concentrer - a été introduite par le cabaliste Isaac Louria (1534-1572) de l'École de Safed, mais elle est déjà présente dans la mystique méditative de l'Espagnol Abraham Aboulafia (1240-1291). «Passé maître dans l'art de l'étude de la signification mystique des lettres et des équivalences de mots en gematria [interprétation fondée sur le calcul des nombres auxquels correspond chaque lettre hébraïque, et qui permet de trouver une équivalence entre les mots dont le total des lettres est identique], il pensait que les différentes combinaisons de lettres et de mots détenaient les forces de création». G. Schoeller, 1993 : 600-605.

14. Le commentaire de Freud sur cet aspect du Dieu schreberien vaut la peine d'être rappelé : «Pour le reproche que Dieu n'apprend rien d'expérience, il est permis de concevoir que nous avons affaire à ce mécanisme du retour à l'envoyeur infantile, qui renvoie sans modification un reproche reçu à celui qui l'a émis». Et Freud ajoute cette note: «Il y a quelque chose d'extraordinairement semblable à une telle revanche lorsque le malade note un jour cette phrase dans ses cahiers: Toute tentative d'action éducative vers l'extérieur doit être abandonnée comme étant sans espoir (188). Celui qui est inéducable, c'est Dieu " (Freud, 1995: 51). Freud rappelle dans cette lecture le rôle du père Schreber, médecin et éducateur.

15. Freud, 1973: 190 (lettre $n^{\circ} 68$ ). Cette théorie a trouvé sa réalisation dans le livre délirant de Fliess, Les Relations entre le nez et les organes génitaux féminins, présentées selon leur significations biologiques, publié à Leipzig et Vienne en 1897 et réédité en français à Paris (Seuil, 1977).

\section{RÉFÉREN CES BIBLIO G RAPHIQ U ES}

ABOUlafiA, A. [1985]: L'Épître des sept voies (préface de S. Trigano), Paris, Éd. de l'Éclat.

Atlan, H. [1982]: "Niveaux de significations et athéisme de l'écriture", La Bible au présent. Actes du colloque des intellectuels juifs de langue française, Paris, Gallimard.

BANON, D. [1987] : La Lecture infinie, Paris, Seuil.

FLIESS [1897] : Les Relations entre le nez et les organes génitaux féminins, présentées selon leurs significations biologiques, réédité en français à Paris (Seuil, 1977).

FREUD, S. [1995] : Le Président Scheber. Remarques psychanalytiques sur un cas de paranoïa, Paris, P.U.F./Quadrige;

[1973]: La Naissance de la psychanalyse, Paris, P.U.F.

IDEL, M. [1989]: L'Expérience mystique d'Abraham Aboulafia, Paris, Cerf; [1998]: La Cabale, Nouvelles perspectives, Paris, Cerf.

LACAN, J. [1975]: Le Séminaire, livre II. Les Psychoses, Paris, Seuil; [1966]: Écrits, Paris, Seuil.

LANGER, G. [1990]: L'Érotique de la kabbale, Paris, Solin.

Melman, C. [1999]: Le Retour à Schreber, Paris, Éd. de l'Association freudienne internationale.

Meschonnic, H. [1973] : Pour la poétique II, Paris, Gallimard.

MANNONI, O. [1969]: Clefs pour l'Imaginaire ou l'Autre Scène, Paris, Seuil, coll. «Points », 75-99.

OUAKNIN, M.-A. [1992]: Tsimtsoum. Introduction à la méditation hébraïque, Paris, Albin-Michel.

Rabant, C. [1978]: Délire et Théorie, Paris, Aubier-Montaigne. SAussure, F. de [1969]: Cours de linguistique générale, Paris, Payot. SCHOELlER, G. (s. la dir.) [1993]: Dictionnaire encyclopédique du judaïsme, Paris, Cerf/Laffont.

Wolf, M.-A. [1998]: Quand le mysticisme mène à la folie, Paris/Montréal, Nadir. 